Volume 15 Issue 1

DOI: http://dx.doi.org/10.53772/NMO.2021.15102

\title{
Future Advancement \\ Potential of Gene editing and RNAi in SARS-Cov-2 diagnosis and therapy
}

\author{
Ashwani Tandon \\ Editor In Chief \\ NMO Journal \\ Journalnmo@gmail.com
}

SARS-CoV-2 surveillance is important to controlling widespread transmission and contains the disease in small peaks. Although mask and vaccination are playing definite role in the prevention of disease but significant work is required in both novel diagnostics and therapeutics. Scientific community is globally working tirelessly to address this gap at the earliest. SARS-CoV-2 is closely related to beta coronavirus species and subspecies; therefore, the detection assays may generate the false-positive results if test are not specifically designed against SARS-CoV-2. Secondly, patient may develop a simultaneous co-infection with other virus. The Confirmation of the COVID-19 is by virus isolation followed by RT-PCR and sequencing. This led to newer classification scheme recently given by WHO to avoid geopolitical controversies and develop consensus. (01).

There is the urgent requirement of novel therapeutic technologies that must be rapid, precise, stable, easy to manufacture, and target-specific for surveillance and treatment. Berber et al beautifully presented the insight on this issue with theoretical clarity and published it in gene therapy. (01) The Advance molecular tools such as gene-editing approaches e.g. CRISPRCas12/13-based SHERLOCK, DETECTR, CARVER and PAC-MAN, antisense oligonucleotides, antisense peptide nucleic acids, ribozymes, aptamers, and RNAi silencing approaches produced with cutting-edge scientific advances. These tools in comparison to conventional diagnostic or therapy may play pivotal role SARS-CoV-2 and other future outbreaks. (01).

\section{Factual status of SARS-Cov-2 and molecular biology tools:}

a. SARS-CoV-2 is an RNA virus and the novel CRISPR can target or diagnose it. 
b. ASO therapy targeting transcript encoding a viral protein or genomic RNA itself could be developed as a response to the SARS-CoV-2 pandemic.

c. Antisense peptide nucleic acids is potential as antiviral agents

d. Although ribozyme and aptamer technologies are relatively older but are still strong candidates in COVID-19 pandemic as it is specific and less toxic, are reproducible.

e. siRNAs that target structural or non-structural proteins of SARS-CoV-2 and may destroy viral assembly.

The SARS-CoV-2 has a RNA genome without segmentation. It encodes four major structural proteins viz. the nucleocapsid $(\mathrm{N})$, the transmembrane $(\mathrm{M})$, the envelope $(\mathrm{E})$, and the spike $(\mathrm{S})$ protein(s). (02-04) The pathophysiological understanding of nonstrctural and structural proteins help us to understand its virulence are potential targets as described above. These toolbox including CRISPR-Cas, antisense oligonucleotides (ASO), peptide nucleic acids (PNAs), ribozymes, and siRNA. ${ }^{(02-06)}$

\section{How CRISPR-Cas help in diagnosis or therapy:}

As we know that the Clustered Regularly Interspaced Short Palindromic Repeats-Cas (CRISPRCas) edit genes and modulate gene expression against both DNA and RNA virus and defend mammalian cell through reprogramming. Cas9 application showed its worth for mammalian cell protection against dsDNA or ssRNA viruses. This open gateway for antiviral therapies. The Cas genes are classified into three main types (I, II, and III) and 11 subtypes (I-A to I-F, II-A to II-C, and III-A to III-B). (07) The Cas futher develop protein moleities in the vicinity of CRISPR to produce CRISPR RNAs (crRNAs).

Cas12a (DNA-specific) and Cas13 (works with RNA) are particularly two popular nucleases in CRISPR diagnostics. In 2017 the SHERLOCK was announced by Feng Zhang's group. It is CRISPR-based nucleic acid detection technique. This strategy can be used in COVID-19 diagnostics. (08) Zhang et al. has developed CRISPR-Cas13 based diagnostics protocol for SARS-CoV-2. ${ }^{(09)}$

\section{How CRISPR-Cas help in antiviral therapy:}

In the human lung epithelial cells the PAC-MAN (prophylactic antiviral CRISPR in human cells), which is a CRISPR-Cas13-based technology can effectively degrade SARS-CoV-2 sequences and live IAV genome. ${ }^{(10)}$ 


\section{ASO as a COVID-19 antiviral therapeutics:}

ASO technology targets mRNA, small RNA, or long non-coding RNA and therefore important in COVID-19. ASO have chemically synthesized 15-50 nucleotides in length that bind RNA targets via complementary base pairing. In the cytosol, the ASO interferes and prevent translation of the mRNA and block protein synthesis; (2) in the nucleus, ASO bind complementary sequence of an mRNA. ${ }^{(11)}$

\section{PNAs as antisense agents:}

Antisense therapy was first described in 1991 and later developed for the treatment of malignant and viral diseases and is characterized by inhibiting gene translation of sequence-specific ASO that binds to target RNA. (12) The stable PNA has lesser binding affinity and this limitation may be overcome by various PNA modifications e.g. it's coupling with gold nanoparticles (AuNPs). After this modification it become less toxic, display high biodistribution and biocompatibility. Its size, shape become adjustable and its synthesis will be easy. ${ }^{(13,14)}$

\section{Use of PNAs in COVID-19}

The strategy that inhibit the viral life cycle at different stages will be important in COVID-19. The use of PNAs is almost unexplored but potential candidate. As we know that the PNA antisense therapeutic approaches have been developed for HIV. In a study, antisense PNAs were used against SARS-CoV which is genetically $79 \%$ similar to SARS-CoV-2 strains. ${ }^{(15)}$

\section{Ribozymes and aptamers in the fight with SARS-CoV-2}

Ribozymes are RNA molecules with catalytic function. This catalytic function is provided by binding and cleavage of the phosphodiester bonds in a target nucleic acid. Ribozyme-based gene regulation studies have been carried out in diseases caused by RNA viruses including HCV, HIV, and influenza. Most of the gene-editing studies carried out by ribozymes had utilized easily modified small ribozymes such as the hammerhead, the hairpin, hepatitis delta, and the Varkud satellite (VS) ribozyme [101,102,103,104,105,106]. The Japanese scientist patent protection by (JP2007043942) the chimeric ribozyme. This ribozyme targets and cuts conserved regions in the coronavirus family and could be adapted to SARS-CoV-2 treatment strategies or studies, as well.

\section{RNAi based approaches for COVID-19 therapy:}

RNA interference (RNAi) mechanism is used for the silencing of genes especially in cancer, viral infections, and autoimmune diseases. There are microRNAs, siRNAs, and shRNAs. These are the key molecules for the activation of RNAi mechanisms. siRNAs, short interfering RNAs, are non-coding dsRNA molecules that are 21-25 bp in length [9, 50, 131,132,133]. siRNAs can be used for the silencing of SARS-CoV-2 genes which encode structural and nsps. Genomes of SARS-CoV-2 encode four structural proteins; these are envelope (E), membrane (M), nucleocapsid $(\mathrm{N})$, and spike $(\mathrm{S})$ proteins which are crucial for the assembly of the virion and inhibition of viral replication in humans. 


\section{Conclusion:}

This review is presenting upcoming strategies for development of novel therapy via gene editing platform. In summary the SARS-Cov-2 virus blamed to be genetically engineered may be treated by gene editing tools in future by global scientific community. Till that time mask, vaccine and physical distancing may act as control measures.

\section{Reference}

1. Berber, B., Aydin, C., Kocabas, F. et al. Gene editing and RNAi approaches for COVID-19 diagnostics and therapeutics. Gene Ther 28, 290-305 (2021).

2. Schoeman D, Fielding BC. Coronavirus envelope protein: current knowledge. Virol J. 2019; 16:69.

3. Hasöksüz M, Kiliç S, Saraç F. Coronaviruses and sars-cov-2. Turkish J Med Sci. 2020;50: $549-56$.

4. Perlman S, Netland J. Coronaviruses post-SARS: update on replication and pathogenesis. Nat Rev Microbiol. 2009; 7: 439-50.

5. Chen Y, Liu Q, Guo D. Emerging coronaviruses: genome structure, replication, and pathogenesis. J Med Virol. 2020c;92: 418-23

6. Hasoksuz M, Sreevatsan S, Cho KO, Hoet AE, Saif LJ. Molecular analysis of the S1 subunit of the spike glycoprotein of respiratory and enteric bovine coronavirus isolates. Virus Res. 2002; 84: 101-9.

7. Jiang F, Doudna JA. The structural biology of CRISPR-Cas systems. Curr Opin Struct Biol. 2015; 30: 100-11

8. Wang Y, Kang H, Liu X, Tong Z. Combination of RT-qPCR testing and clinical features for diagnosis of COVID-19 facilitates management of SARS-CoV-2 outbreak. J Med Virol. 2020c; 92:538-9.

9. Metsky HC, Freije CA, Kosoko-Thoroddsen T-SF, Sabeti PC, Myhrvold C. CRISPR-based surveillance for COVID-19 using genomically-comprehensive machine learning design. BioRxiv. 2020.

10. Abbott TR, Dhamdhere G, Liu Y, Lin X, Goudy LE, Zeng L, et al. Development of CRISPR as a prophylactic strategy to combat novel coronavirus and influenza. BioRxiv. 2020; 1-20.

11. Schubert S, Kurreck J. Oligonucleotide-based antiviral strategies. Handb Exp Pharmacol. 2006.

12. Galderisi U, Cascino A, Giordano A. Antisense oligonucleotides as therapeutic agents. J Cell Physiol. 1999; 181: 251-7. 
13. Ghaffari E, Rezatofighi SE, Ardakani MR, Rastegarzadeh S. Delivery of antisense peptide nucleic acid by gold nanoparticles for the inhibition of virus replication. Nanomedicine. 2019.

14. Su X, Kanjanawarut R. Control of metal nanoparticles aggregation and dispersion by PNA and PNA-DNA complexes, and its application for colorimetric DNA detection. ACS Nano. 2009.

15. Lu R, Zhao X, Li J, Niu P, Yang B, Wu H, et al. Genomic characterisation and epidemiology of 2019 novel coronavirus: implications for virus origins and receptor binding. Lancet. 2020.

How to Cite this article As: Tandon, A. (2021). Future Advancement: Potential of Gene editing and RNAi in SARSCov-2 diagnosis and therapy National Medicos Organisation Journal (एन एम ओ जर्नल) (ISSN-2348-3806), 15(1), 03-07. Retrieved from https://nmojournal.org/index.php/nmojournal/index 